\title{
The Effects of Exercise in Obese Women with Bulimia Nervosa
}

\author{
Nasim Habibzadeh*, MSc; Hassn Daneshmandi, PhD
}

\author{
Authors' Affiliation: \\ Department of Sport Sciences, \\ University of Guilan, Rasht, \\ IR Iran

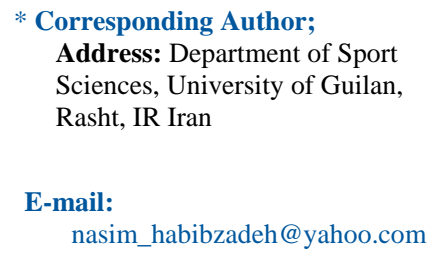

Received: Aug 30, 2010

Accepted: Nov 16, 2010

Key Words: Bulimia Nervosa; Obese; Women; Walking; Exercise

\begin{abstract}
Purpose: Obesity has been identified as a risk factor for the development of bulimia nervosa (BN) in those who try to lose weight. The purpose of the present study was to examine the effect of walking exercise in order to provide a method for overcoming bulimia nervosa in obese young women suffering from bulimia nervosa.

Methods: Twenty obese women with bulimia nervosa (body mass index $[\mathrm{BMI}]>30$ ) and a mean age of $22.00 \pm 1.50$ years volunteered to participate in this study. They were randomly assigned to exercise $(n=10)$ and control $(n=10)$ groups. Both groups underwent anthropometric measurements and blood analysis before and after the training program. Exercise program included 30-minute walking sessions at $50-75 \%$ of maximal heart rate, 3 days per week and for 2 months.
\end{abstract}

Results: After 2 months significant changes were observed in all anthropometric variables $(P<0.001)$. Percent body fat, fat mass, BMI, body weight and lean mass changes in response to training were significant in the exercise group $(P<0.001)$.

Conclusion: This study demonstrated that moderate aerobic exercises such as moderate walking are suitable behavior therapies for overcoming bulimia nervosa in obese young women.

\section{INTRODUCTION}

Eating disorders and obesity are parts of a range of weight-related problems. Body dissatisfaction and unhealthy dieting practices are linked to the development of eating disorders, obesity and other problems ${ }^{[1]}$. Obesity has been identified as a risk factor for the development of bulimia nervosa (BN), and mild, moderate and severe obesity have repeatedly been observed in these patients ${ }^{[2]}$. It has previously been stated that individuals with $\mathrm{BN}$ are typically within the normal weight range, although some may be slightly underweight or overweight. However, it has been suggested that prior to the onset of $\mathrm{BN}$, individuals with this eating disorder are more likely to be overweight than their peers ${ }^{[3]}$. The eating disorders such as anorexia nervosa and bulimia nervosa and the condition of obesity have been known since earliest times in Western civilization ${ }^{[4]}$. Bulimia nervosa can be extremely harmful to the body. The recurrent binge- 
and-purge cycles can damage the entire digestive system and purge behaviors can lead to electrolyte and chemical imbalances in the body that might affect the functions of heart and other major organs ${ }^{[5]}$. Adolescent girls may suffer from more than one disorder or may progress from one problem to another at varying degrees of severity ${ }^{[6]}$. It is important to understand this range of weight related problems in order to avoid causing one disorder, such as bulimia, while trying to prevent another, such as obesity ${ }^{[7]}$. Patients can develop bulimia at any age from the preteens to the 50's with the peak onset at 18-20 year of age. Obese people were punished for their adiposity particularly youths who gained weight were subjected to compulsory diets and scourging. Because of all the negative physiologic and psychosocial consequences associated with an excessive amount of body fat, obese people frequently try to lose weight ${ }^{[8]}$. Although eating and weight disorders are common in children and adolescents, there is a scarcity of practical treatment methods for eating disorders in young people ${ }^{[9]}$. The best treatment for obesity is weight control in a good manner. Behavior therapies provide methods for overcoming barriers to compliance with dietary therapy and/or increased physical activity, and are important components of weight loss therapy ${ }^{[8]}$. While various forms of physical activity may cause slow loss of obesity, walking as an exercise intervention may be accepted by a board range of patients. There may be a therapeutic role for walking exercise in the improvement and management of bulimia nervosa in obese persons. Furthermore, it is popular and feasible for the obese population ${ }^{[10]}$. Previous studies have found that losing even 10 lbs. makes a significant difference contributing to overall health and wellbeing, even when subjects are still obese ${ }^{[11]}$. Research shows that overweight bulimic girls are more concerned about their weight, more dissatisfied with their bodies and more likely to go on a diet than their normal-weight peers ${ }^{[12]}$. High numbers of adolescent girls report that they are dissatisfied with their bodies and try to lose weight in unhealthy ways including skipping meals, fasting and using tobacco. At the same time, more active leisure time activities are getting replaced by low activity hobbies such as watching television and working with computer ${ }^{[13]}$. As obesity increases all- cause mortality risk this should be considered attentively. Besides, it has been shown that obesity in youth is a more powerful predictor of bulimia nervosa than in adulthood. The purpose of the present study was to examine the effect of walking exercise in order to provide a method for overcoming bulimia nervosa in young obese women.

\section{METHODS AND SUBJECTS}

Subjects were recruited through various advertising strategies such as posters in physicians' offices, daycare centers and drugstores. The initial diagnosis of bulimia nervosa was made by a competent medical professional. The DSM-IV criteria were used to diagnose bulimia nervosa in this study ${ }^{[14]}$. Twenty untrained bulimic obese body mass index $(\mathrm{BMI})>30$ women with a mean age of $22.00 \pm 1.50$ years volunteered to participate. They were randomly assigned to exercise and control groups (Experimental $n=10$, Control $n=10$ ). Written informed consent for all procedures was obtained from all participants prior to entering the study. The criteria for the invitation were evaluating by questionnaire including willing to participate, being clinically healthy (no cardiovascular, musculoskeletal, respiratory or other chronic diseases that could limit training or testing), no menstrual irregularities, using no medication and no betablockers, sedentary life style, non-dieting and no apparent occupational or leisure time responsibilities that could impede their participation. The following measurements were made at baseline prior to the start of the exercise program and after completion of the 2month training program. This study was approved by local committee of ethics.

\section{Dietary Intake}

Caloric expenditure was calculated based on the weight of the subject via the Harris-Benedict formula based on total body weight $[\mathrm{BMR}=655+(9.6 \mathrm{X}$ wt in $\mathrm{kg})+$ (1.8 X ht in $\mathrm{cm})$ - (4.7 $\mathrm{X}$ age in years)]. To minimize any effect that dietary composition might have on the measured metabolic variables, all subjects were 
instructed on the American Health Association (AHA) diet by registered dietitian at the initiation of the study. We gave a diet program with equal calorie to exercise group, which was about $2400 \mathrm{kcal}$ a day. The composition of this diet was carbohydrate (50-55\%), protein $(15-20 \%)$ and fat $(<30 \%)^{[15]}$.The subjects were asked to maintain this diet throughout the study (2months). Compliance was monitored by review of 7day food records taken every week.

\section{Anthropometric measurement}

Body weight and height were recorded and BMI was calculated as weight (kg) divided by height (m) squared. Fat mass, percent body fat and lean mass were assessed with bioelectrical impedance equipment (BIA106, RJL Systems, USA). In addition, all subjects were weighed every week. A physician recognized the commonly coexisting psychiatric conditions such a both cognitive behavioral psychotherapy and interpersonal psychotherapy in exercise subjects every two week.

\section{Exercise program}

The program included warming-up phase for 5 minutes of stretching exercises, 30 minutes of walking at 50$75 \%$ of maximum heart rate and cooling-down phase for 5 minutes of stretching, three times a week for 2 months. Stretching exercises were performed for the arms, leg, back and stomach. A target heart rate ranging between $50-75 \%$ of age adjusted maximum heart rate intensity was calculated for each walker from her age and walking supine resting heart rate ${ }^{[16]}$. Heart rate was measured with an electronic heart rate meter (Sport Tester PE, Polar Electro, Finland). The exercise program was accompanied by music. All sessions were supervised by a professional exercise physiologist leader.

\section{Statistical Analysis}

The data were analyzed using the SPSS statistical package (SPSS 13 for Windows; SPSS, Chicago, USA). Mean and standard deviation (SD) were used as descriptive statistics. Student's t-test was used for normally distributed variables. Unpaired t-test was used to assess the change in anthropometric variables level of significance was accepted as $P<0.05$ for all comparisons.

\section{RESULTS}

Twenty subjects (100\%) completed the training program. No major change in menstrual status was observed during the study. Table 1 shows the physical characteristics of the study subjects (pre, post study). There were no significant differences in mean age, height and BMI between the two groups before the study. Percent body fat (2.2\%), fat mass (2\%) and lean mass $(1.1 \%)$ changes in response to training were significant in the exercise group. The lean mass in exercise group significantly increased but the present

Table 1: Changes in Anthropometric variables pre- and post-exercise (X $\pm S D)$

\begin{tabular}{lccccc} 
Variable & \multicolumn{2}{c}{ Obese (Experimental) } & \multicolumn{2}{c}{ Obese (Control) } & \multirow{2}{*}{ P value } \\
\cline { 2 - 5 } Age (year) & Pre & Post & Pre & Post & \\
Height (cm) & $22.22 \pm 1.98$ & - & $22.67 \pm 1.50$ & - & \\
Weight (kg) & $157.78 \pm 5.11$ & - & $159.11 \pm 1.50$ & - & $<0.001$ \\
BMI (kg/m $\mathbf{2}^{2}$ & $74.98 \pm 8.11$ & $73.27 \pm 7.74$ & $78.11 \pm 10.88$ & $78.06 \pm 10.14$ & $<0.001$ \\
Lean mass (kg) & $30.20 \pm 1.83$ & $28.88 \pm 2.10$ & $30.93 \pm 3.57$ & $30.41 \pm 3.05$ & $<0.001$ \\
Fat mass (kg) & $43.27 \pm 5.25$ & $44.38 \pm 6.21$ & $43.86 \pm 6.03$ & $43.25 \pm 6.67$ & $<0.001$ \\
\% Body fat & $29.11 \pm 4.54$ & $27.17 \pm 6.30$ & $31.16 \pm 6.28$ & $31.42 \pm 7.13$ & $<0.001$
\end{tabular}


body fat and fat mass decreased significantly (all $P<0.001)$. The mean body weight was $1.3 \%$ lower at the end of the study. Body mass index (BMI) in the exercise group (2.3\%) significantly differed from before the intervention compared with the control group $(P<0.001)$.

\section{DISCUSSION}

Mildly to moderately overweight patients may need treatment only if their weight is causing or worsening other medical conditions, and are generally benefited from increased physical activity and decreased fat consumption without the chronic hunger of dieting. Regular moderate exercise is helpful in both managing stress and promoting a healthy body shape and composition in individuals with bulimia nervosa. In our patients a simple 30 minutes of walking exercise at the range of $50-75 \%$ of maximum heart rate was enough to decrease the percent body fat, fat mass, BMI, body weight and increase lean body weight. Exercise does more than just get the muscles working to burn calories and burn fat. It helps to speed up the metabolism ${ }^{[17]}$. Thompson et al also indicated an inverse association between body composition and daily walking exercise in middle-aged obese women. Walking may not involve high loading but it is accessible to majority of women, in particular women with eating disorders ${ }^{[18]}$. Researches showed that weight bearing and muscle contractions generate stress on lean body necessary to prevent lose weight. "Lean mass", the researchers conclude, "is the major determinant of body size, providing further evidence that body size is adapted to the dynamic load imposed by muscle force rather than passive loading by fat" ${ }^{[19]}$. This issue is desirable for obese women with bulimia nervosa because they do not like any excessive fat mass in their body, and they continuously try to lose weight ${ }^{[20]}$. Our findings reveal that 2-month walking exercise is of sufficient duration and intensity to result in significant improvements in all components of body composition in obese women. The decrease in body weight in exercise group accounts for the responses of body weight to walking exercise compared with the control group. Fenkci et al have shown that a 12-week aerobic exercise decrease BMI, waist and weight measurements significantly. Consequently, they have indicated that aerobic exercise induces improvement in body fat composition and has a favorable metabolic effect in obese women with severe eating disorders ${ }^{[21]}$. In contrast exercise programs for weight loss are not always successful because of dropout rates, complaints of discomfort during exercise and inability to control participants' eating during the program. Furthermore, achieving weight loss in overweight and obese individuals is difficult, particularly in the presence of anxiety and depression ${ }^{[22]}$. Levine et al demonstrated that a 6month exercise intervention in obese women with binge eating disorder did not significantly improve symptomatology of eating disorders in comparison with control subjects ${ }^{[23]}$. It may be related to duration of exercise in this study in obese women.

\section{CONCLUSION}

The present study concluded that there is the need to aggressively promote lifestyle intervention, especially exercise. Although our study population was small, moderate physical activity was shown to be associated with weight loss and healthier body in obese women with bulimia nervosa. It seems that moderate aerobic exercises such as walking are suitable behavior therapies for overcoming bulimia nervosa in obese young women.

\section{ACKNOWLEDGMENTS}

The work was supported by University of Guilan. We would like to acknowledge our colleague Dr. Mohsen Madah, who assisted in diagnosis of bulimia nervosa in our participants. We also gratefully acknowledge all subjects who cooperated in this investigation.

Conflict of interests: None 


\section{REFERENCES}

1. Mitchell JE, Pyle RL, Eckert ED, Hatsukami D, Soll E. Bulimia nervosa in overweight individuals. J Nerv Ment Dis. 1990;178:324-7.

2. Fairburn CG, Welch SL, Doll HA, Davies BA, O'Connor ME. Risk factors for bulimia nervosa. A community-based casecontrol study. Arch Gen Psychiatry. 1997;54:509-17.

3. Neumark-Sztainer D. Obesity and Eating Disorder Prevention: An Integrated Approach. Adolesc Med. 2003;14:159-73 .

4. Wittchen HU, Nelson CB, Lachner G. Prevalence of mental disorders and psychosocial impairments in adolescent and young adults. Psychol Med. 1998;28:109-26.

5. Garner DM, Wooley SC. Confronting the failure of behavioral and dietary treatments for obesity. Clin Psychol Rev. 1991;11:729-80.

6. Rosen JC, Reiter J. Development of the Body Dysmorphic Disorder Examination. Behav Res Ther 1996;34:755-66.

7. Presnell K, Stice E. An experimental test of the effect of weight-loss dieting on bulimic pathology: tipping the scales in a different direction. J Abnorm Psychol. 2003;122:166-70.

8. Wadden TA, Foster GD. Behavioral treatment of obesity. Med Clin North Am. 2000;84:441-61.

9. Fairburn CG, Cooper Z, Doll HA, Norman P, O'Connor M. The natural course of bulimia nervosa and binge eating disorder in young women. Arch Gen Psychiatry. 2000;57:659-65.

10. Klem LM, Wing RR, McGuire TM, Seagle MH, Hill OJ. A descriptive study of individuals successful at long-term maintenance of substantial weight loss. Am J Clin Nutr.1997;66:239-46.

11. Chen KY, Acra SA, Donahue CL, Sun M, Buchowski MS. Efficiency of Walking and Stepping: Relationship to Body Fatness. Obes Res. 2004;12:982-9.

12. Rosen JC, Orosan P, Reiter J. Cognitive behavior therapy for negative body image in obese women. Behav Ther. 1995;26:2542.

13. Wadden TA, Brownell KD, Foster GD. Obesity: responding to the global epidemic. J Consult Clin Psychol. 2002;70:510-25.

14. American Psychiatric Association. Diagnostic and Statistical Manual of Mental Disorders (4 ${ }^{\text {th }}$ ed.) United States of America: American Psychiatric Association, 1994.

15. New SA, Bolton-Smith C, Grubb DA, Reid DM. Nutritional influence on bone mineral density: a cross sectional study in premenopausal women. AM J Clin Nutr. 1997;65:1831-9.

16. Karvonen J, Vuorimaa T. Heart rate and exercise intensity during sport activities: Practical application. Sport Med. 1988;5: 303-11.

17. Caplan GA, Ward JA, Lord SR. The benefits of exercise in premenopausal women. Aust J Public Health.1999;17:23-6.

18. Thompson LD, Rakow J, Perdue MS. Relationship between accumulated walking and body composition in middle-aged women. Med Sci Sports Exerc. 2004;36:911-4.

19. LaValle JB, Krinsky DL, Hawkins EB, Pelton R, Ashbrook Willis N. Natural Therapeutics Pocket Guide. Ohio: Lexi Comp. 2000; Pp:387-8.

20. Field T, Schanberg S, Kuhn C, et al. Bulimic adolescents benefit from massage therapy. Adolescence. 1998;33:555-63.

21. Fenkci S, Sarsan A, Rota S, Ardic F. Effects of resistance or aerobic exercises on metabolic parameters in obese women who are not on a diet. Department of Internal Medicine, Division of Endocrinology and Metabolism, Turkey. 2006;23:404-13.

22. Ross C. Overweight and depression. J Health Soc Behav.1994:35:63-79.

23. Levine MD, Marcus MD, Moulton P. Exercise in the treatment of binge eating disorder. Int J Eating Disorder. 1996;19:171-7. 